\title{
Weakly bound and unbound nuclei near the neutron drip line
}

\section{Takashi Nakamura*}

Dept. of Physics, Tokyo Institute of Technology

E-mail: nakamuraephys.titech.ac.jp

\begin{abstract}
Recent experimental studies on exotic nuclei near and beyond the neutron drip line using the multi-purpose large acceptance spectrometer SAMURAI at RIBF at RIKEN are presented. In particular, we focus on the experiment on the beyond-drip-line nucleus ${ }^{26} \mathrm{O}$ produced by the oneproton removal reaction of ${ }^{27} \mathrm{~F}$ at $201 \mathrm{MeV} /$ nucleon. We have found that the ground state is barely unbound, only $18 \pm 3$ (stat.) \pm 4 (syst) $\mathrm{keV}$, with respect to the two neutron emission threshold. We discuss the implication of such a finding. We also show perspectives on the on-going and near future projects at SAMURAI on the neutron drip line nuclei.
\end{abstract}

The 26th International Nuclear Physics Conference

11-16 September, 2016

Adelaide, Australia

${ }^{*}$ Speaker. 


\section{Introduction}

One of the interesting features of nuclei in the vicinity of the drip lines, the bound limits of atomic nuclei, is possible enhancement of the nucleon-nucleon correlations. Dobaczewski et al. suggested that a strong pairing correlation occurs for a weakly-bound nucleus when the absolute value of the separation energy becomes significantly smaller than the paring gap $\Delta$, i.e., $\left|S_{\mathrm{n}}\right| \ll \Delta[1]$. A spatially compact $n n$ correlation, called dineutron, is one of such cases. The dineutron was predicted originally by Migdal more than four decades ago [2] as a strongly spatially correlated two neutron system on the surface of a nucleus, although such a correlation has never been observed directly. More recently, the dineutron correlation was theoretically studied for low-dense neutron matter [3, 4], which suggests that neutron halo and skin could exhibit dineutron correlations. Experimentally, the dineutron has been investigated for the light halo nuclei such as ${ }^{11} \mathrm{Li}$ where the dineutron correlation was indeed suggested by the enhancement of the electric dipole strength [5]. However, this result assumed that the ${ }^{11} \mathrm{Li}$ nucleus has a pure ${ }^{9} \mathrm{Li}+n+n$ configuration, and had some model dependence about the radial size of the halo distribution. Hence, this is considered as an indication, rather than the evidence of dineutron. Hence, it is important to study further the dineutron correlations along the neutron drip line.

Compared to the proton drip line, nuclei along the neutron drip line tend to show more novel nuclear structure as the latter has larger neutron/proton asymmetry. The neutron halo structure is more developed, compared to the proton halo, due to no Coulomb barrier. On the other hand, studies of neutron drip line nuclei are more challenging as it is farther from the $\beta$ stability than the proton drip line. As shown in Fig. 1, the neutron drip line has been established experimentally only up to $Z=8$ at ${ }^{24} \mathrm{O}$. Interestingly, the neutron drip line is stalled at $N=16$ for $Z=6-8$, while it is extended at least $N=22$ for fluorine $(Z=9)$. This sudden leap of the neutron drip line has not yet been understood (oxygen drip-line anomaly) [6]. This region of drip line is also interesting in terms of the shell evolution since the island of inversion near $Z=10-12$ and $N=19-22$ is close by. Recently, the deformation-driven $p$-wave halo configuration has been found for ${ }^{29} \mathrm{Ne}$ [7], ${ }^{31} \mathrm{Ne}$ [8, 9], and ${ }^{37} \mathrm{Mg}$ [10], using the nuclear and Coulomb breakup at 230-240 MeV/nucleon. The large radii of these nuclei, consistent with such halo structure, were shown by the enhanced reaction cross sections [11, 12]. Note that these nuclei are on the edge of the neutron drip line as they have the least neutron number in the respective isotonic chains, as shown in Fig. 11

Here, the recent studies on the neutron drip line nuclei using the SAMURAI (Superconducting Analyzer for MUlti-particle from RAdio Isotope Beam) facility [13] at RIBF, RIKEN, are shown. In particular, we focus on the results of heavy oxygen isotope ${ }^{26} \mathrm{O}$ beyond the neutron drip line, which may provide a clue to solve the oxygen drip-line anomaly. Some perspectives for the near future are also shown.

\section{Breakup reactions of exotic nuclei at intermediate energies}

Breakup reactions at intermediate energies have played significant roles in probing nuclei near the drip line. Such nuclei are generally weakly bound, so that the breakup reactions are likely to have larger cross sections. This is advantageous when an RI beam of drip-line nuclei is used where the beam intensity is rather weak. 


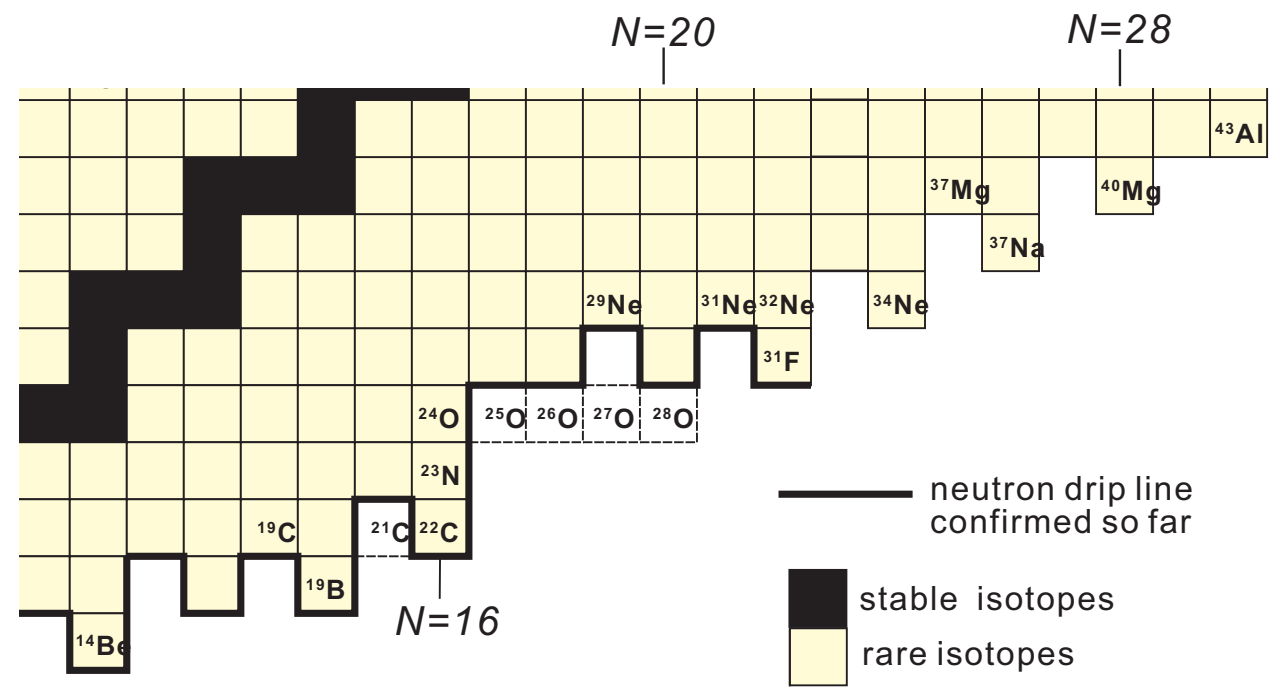

Figure 1: (color online). The neutron drip-line region of the nuclear chart for $5 \leq Z \leq 12$. The drip line has been confirmed experimentally up to $Z=8$. There is a sudden gap of the neutron drip line between $Z=8$, and 9, called oxygen drip-line anomaly.

Nuclear breakup of drip-line nuclei with a light target, such as a carbon target, can probe their single particle states. For instance, when one neutron removal reaction is applied to the one-neutron halo nucleus ${ }^{31} \mathrm{Ne}$, one can extract the information on the angular momentum of the removed neutron, and the single-particle configurations [9]. So that the $p$-wave nature of the ground state, relevant to the neutron halo, could be revealed. When this is applied to the two neutron halo nucleus, for instance ${ }^{22} \mathrm{C}$, then one-neutron removal channel leads to unbound intermediate states of ${ }^{21} \mathrm{C}$. The invariant mass spectroscopy can be used to determine the energy (mass) of ${ }^{21} \mathrm{C}$ by measuring the momenta of ${ }^{20} \mathrm{C}$ and the neutron in coincidence. When we measure the momentum distribution of ${ }^{21} \mathrm{C}$, namely that of the ${ }^{20} \mathrm{C}+n$ system, one can obtain the information of the angular momentum of the removed neutron in ${ }^{22} \mathrm{C}$. One can also study the ${ }^{22} \mathrm{C}$ excited states above the two-neutron decay threshold by examining the inelastic scattering channel, where the ${ }^{22} \mathrm{C}$ energy (mass) can be deduced from the invariant mass reconstructed from all the momentum vectors of the three decay particles, ${ }^{20} \mathrm{C}$ and the two neutrons.

One important application of the nuclear breakup reactions is to produce the unbound nuclei along and beyond the neutron drip line. In fact, quite a number of nuclei beyond the drip line have been observed [14]. Below we demonstrate here the results of such a case on heavy oxygen isotopes beyond the neutron drip line.

Coulomb breakup of halo nuclei with a heavy target, such as $\mathrm{Pb}$, has been used to study the $E 1$ response of such nuclei. For halo nuclei, it has been established that the enhanced $E 1$ strength appears at low excitation energies of about $1 \mathrm{MeV}$, called soft $E 1$ excitation [15]. The energy spectrum of the soft $E 1$ excitation can be used to extract the configuration of the single particle halo states, such as ${ }^{11} \mathrm{Be}[16,17,18],{ }^{15} \mathrm{C}[19]$ and ${ }^{19} \mathrm{C}[20]$, and the two neutron correlation as studied for ${ }^{11} \mathrm{Li}[5]$. 


\section{Breakup reaction experiments at SAMURAI facility}

With the advent of the new-generation RI beam facility, represented by RIBF at RIKEN, the breakup reactions of drip-line nuclei are now measured more efficiently, combining the higherintense, more exotic RI beams with a state-of-the-art spectrometer. As such, the SAMURAI has been commissioned in 2012 as one of key spectrometers at RIBF. The SAMURAI facility is equipped with a large-gap $(80 \mathrm{~cm})$ superconducting magnet with the maximum field of $3.1 \mathrm{~T}$, beam detectors (two drift chambers, an ionization chamber, and trigger scintillators), charged fragment detectors (two drift chambers, and hodoscopes), and neutron detectors NEBULA. The details of the standard detectors used in SAMURAI can be seen in Refs [13,21].

SAMURAI involves several important features: 1 ) large momentum acceptance which allows measurements of a wide-range of outgoing charged particles with $1 \leq A / Z \leq \sim 3$ in one setting, 2) large angular acceptance (about \pm 8.8 degrees in the horizontal direction and about \pm 4.4 degrees in the vertical direction), which is realized by the large gap of the magnet as well as the large area neutron detector array NEBULA, and 3) relatively good momentum resolution of $\Delta P / P \sim 1000$, which is sufficient for the mass separation even with $A \sim 100$.

\section{Day-one campaign experiments at SAMURAI}

Spectroscopy of neutron drip line nuclei using the breakup reactions is one of the important physics programs at SAMURAI. As such, using the ${ }^{48} \mathrm{Ca}$ primary beam at $345 \mathrm{MeV} /$ nucleon, dripline nuclei with $5 \leq Z \leq 10$ have been produced as a secondary beam to study 1) Coulomb breakup of ${ }^{19} \mathrm{~B}$ and ${ }^{22} \mathrm{C}$ (Spokesperson T. Nakamura), 2) structure of unbound nuclei ${ }^{18} \mathrm{~B}$ and ${ }^{21} \mathrm{C}$, and the low-lying excited states of ${ }^{19} \mathrm{~B}$ and ${ }^{22} \mathrm{C}$ (Spokesperson N.A. Orr, and G. Jibelin), and 3) structure of heavy oxygen isotopes ${ }^{25,26} \mathrm{O}$ beyond the neutron drip line (Spokesperson Y. Kondo), whose details are presented in the next section. In this campaign, for instance, the ${ }^{22} \mathrm{C}$ secondary beam impinged on a $\mathrm{Pb}$ target at $235 \mathrm{MeV} /$ nucleon to induce the Coulomb breakup, while that on a $\mathrm{C}$ target to induce the nuclear breakup to produce ${ }^{21} \mathrm{C}$ and the excited states of ${ }^{22} \mathrm{C}$. Note that ${ }^{22} \mathrm{C}$ has drawn much attention recently since it may have a large neutron halo [22, 23].

One of the advantages of this type of experiment is that a variety of reaction channels can be studied in the same setup due to the large momentum acceptance. A cocktail beam used here even enhances the combinations of projectiles and ejectiles. For instance, one can obtain the interaction cross section by measuring the transmission of a specific isotope through a target. In this experiment, we indeed extracted the interaction cross section of ${ }^{22} \mathrm{C}$ on a carbon target, to update the r.m.s. radius of ${ }^{22} \mathrm{C}$ [24]. We also studied the one neutron removal reaction of ${ }^{20} \mathrm{C}$ to produce the excited unbound states of the one-neutron halo nucleus ${ }^{19} \mathrm{C}$, where new states have been observed [25].

\section{Spectroscopy of oxygen isotopes beyond the neutron drip line}

We now discuss the results of ${ }^{26} \mathrm{O}$. The first pioneering work on ${ }^{26} \mathrm{O}$ was performed at MSU using the one proton removal of ${ }^{26} \mathrm{~F}$ at $82 \mathrm{MeV} /$ nucleon, which could locate the ground state at $150_{-150}^{+50} \mathrm{keV}$ above the $2 n$ emission threshold. Interestingly, this state cannot decay by the oneneutron emission, since the ground state of ${ }^{25} \mathrm{O}$ is located at $770_{-10}^{+20} \mathrm{keV}$ [27]. Such a weakly 
"unbound" ground state of ${ }^{26} \mathrm{O}$ was confirmed later by an experiment at GSI [28], which also claimed the finding of the excited state at $4.2 \mathrm{MeV}$. Note that in these experiments only an upper limit was extracted for the ground state energy.

This nucleus has also drawn attention due to the possible two-neutron radioactivity (longlived two neutron emitter). The pioneering theoretical work by Grigorenko [29] suggested that ${ }^{26} \mathrm{O}$ can live long due to the centrifugal barrier of the $d$-wave neutrons if ${ }^{26} \mathrm{O}$ is described by the pure $\left(v 0 d_{3 / 2}\right)^{2}$ upon the doubly-magic ${ }^{24} \mathrm{O}$ core. Kohley et al. at MSU introduced a novel analysis method to extract the life time using the velocity difference between ${ }^{24} \mathrm{O}$ and the neutron in the decay of ${ }^{26} \mathrm{O}\left[30\right.$. Depending on the life time, ${ }^{26} \mathrm{O}$ can decay inside the target or after the target. Because of the different energy loss depending on the breakup point, this velocity difference has a sensitivity to the life time. Accordingly, they evaluated the half life of ${ }^{26} \mathrm{O}$ to be $4.5_{-1.5}^{+1.1}$ (stat) \pm 3 (syst) ps. Although the large systematic error could not allow the definitive conclusion, they claimed that this observation suggests the two-neutron radioactivity. Further theoretical study suggests, however, that even a slight mixture of low- $\ell$ configuration may shorten drastically the life time [31]. As such, more precise extraction of the energy of the state is called for.

In the day-one campaign at SAMURAI, we used one-proton removal of ${ }^{27} \mathrm{~F}$ on a carbon target at $201 \mathrm{MeV} /$ nucleon to produce ${ }^{26} \mathrm{O}$. The details of the experimental conditions and the analysis procedure is seen in Ref. [32]. Figure 2] shows the obtained decay energy spectrum of ${ }^{26} \mathrm{O}$ into ${ }^{24} \mathrm{O}+n+n$. We observed clearly the ground state just above the two neutron decay threshold, as well as an excited state around $1.28 \mathrm{MeV}$. The latter was observed for the first time in this experiment. With a careful analysis shown in Ref. [32], including that on the ${ }^{24} \mathrm{O}+n$ spectrum, we could determine the ground state energy as a finite value of $18 \pm 3$ (stat) \pm 4 (syst) keV. The energy of the first excited state was determined to be $1.28_{-0.08}^{+0.11} \mathrm{MeV}$. In the day-one campaign experiment, we also measured ${ }^{25} \mathrm{O}$ using the one proton removal of ${ }^{26} \mathrm{~F}$. Owing to much higher statistics than the previous experiments [27,28], we could determine precisely the energy and the width of this state as $749 \pm 10 \mathrm{keV}$ and $88 \pm 6 \mathrm{keV}$, respectively. The resultant level scheme thus obtained is shown in Fig. 3 .

The energy of the ${ }^{26} \mathrm{O}$ ground state with respect to the $2 n$ emission is lowest among any other two-neutron emitter that has ever been observed. Such a barely unbound state has two significant points: 1) The life time could be long, and 2) there could exist a strong $n n$ correlation. For the first point, the observed energy of the ground state, compared to the theoretical calculation by Grigorenko et al [31], corresponds to the half-life of the order of $10^{-17}-10^{-15} \mathrm{~s}$. This is much shorter than the evaluation by Kohley et al. [30], of the order of ps, but much longer than the typical decay time relevant to strong interactions $\left(10^{-22} \mathrm{~s}\right)$. Since this life-time estimation is model dependent, further experimental efforts to measure directly the life-time is desired.

The second point on the strong $n n$ correlation is suggested in the idea by Dobaczewski mentioned above [1], where not only weakly bound nuclei but also weakly "unbound" nuclei can have a strong $n n$ correlation. Interestingly, Hagino and Sagawa applied the three-body model to ${ }^{26} \mathrm{O}$ $\left({ }^{24} \mathrm{O}+n+n\right)$ to show a notable dineutron structure in this nucleus [33, 34]. They also showed that, due to the dineutron in ${ }^{26} \mathrm{O}$, in the 3-body decay of this nucleus, the two neutron should be emitted back-to-back [33, 34]. This can be understood naively by the fact that a smaller distance between the two neutrons in the dineutron corresponds to a larger relative momentum between the two neutrons as a result of the Heisenberg's uncertainty principle. A pioneering experimental attempt to 


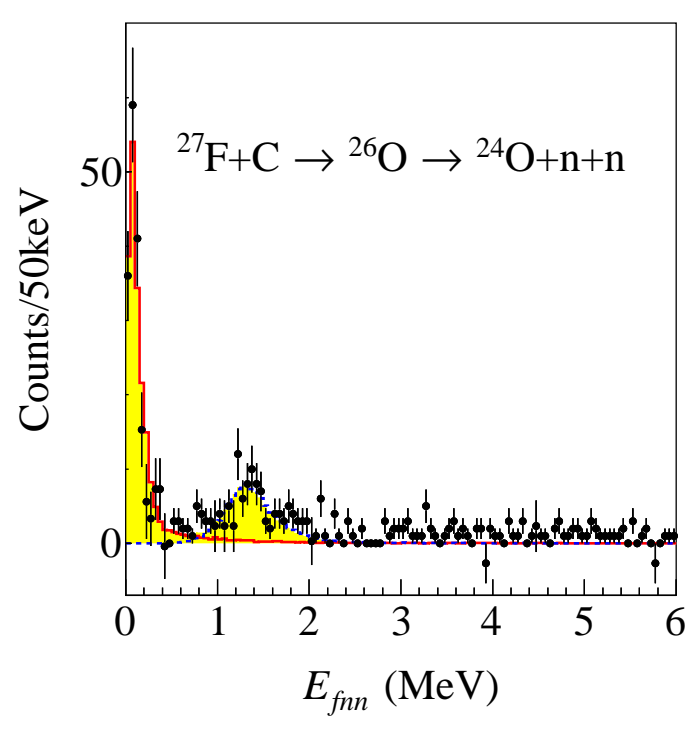

Figure 2: (color online). The decay energy spectrum of ${ }^{26} \mathrm{O}$ into ${ }^{24} \mathrm{O}+n+n$, obtained in the proton removal reaction of ${ }^{27} \mathrm{~F}$ at $201 \mathrm{MeV} /$ nucleon [32].

\section{$\underline{E x=1.28^{+0.11}{ }_{-0.08} \mathrm{MeV}}$}

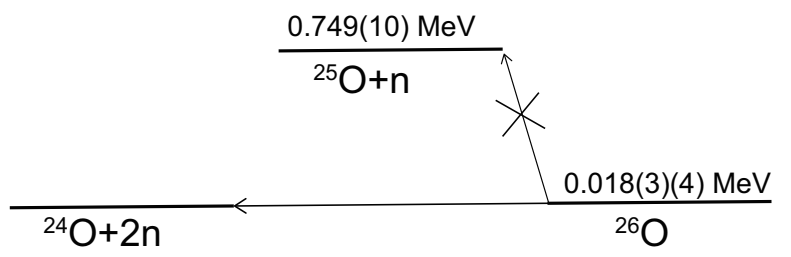

Figure 3: The level scheme of ${ }^{25} \mathrm{O}$ and ${ }^{26} \mathrm{O}$ obtained in the proton removal reaction of ${ }^{26} \mathrm{~F}$ and ${ }^{27} \mathrm{~F}$, respectively, at SAMURAI at RIBF [32].

search for such angular correlation at MSU, however, failed due to the insufficient resolutions of the experimental setup [35]. Much higher angular resolution is required for such a measurement, so even the current SAMURAI setup would not be able to obtain the angular correlation signal. We thus require a new detection scheme for the three-particle coincidence in the future.

Most of the traditional theories could not predict well the location of the oxygen drip line (oxygen drip-line anomaly). One solution would be the three-nucleon force effects as proposed first by Otsuka et al. [36]. The work by Hergert et al. [37] is the ab-initio type study including the threenucleon forces. The other notable theoretical work on ${ }^{26} \mathrm{O}$ was made by Volya and Zelevinsky, where continuum shell model was applied [38]. They predicted the energy of the ground state of ${ }^{26} \mathrm{O}$ as $21 \mathrm{keV}$, in remarkably agreement with the current experimental observation. This may suggest that the effects of continuum is indeed important in the nuclei beyond the drip line. In any cases, our current results on the ground state and the first excited state of ${ }^{26} \mathrm{O}$ certainly put strong 
constrains on theoretical calculations.

\section{Summary and Perspectives}

This presentation showed recent experimental studies on the structures of nuclei near and beyond the neutron drip line using the breakup at the SAMURAI facility at RIBF at RIKEN. Nuclear and Coulomb breakup have played significant roles in probing such nuclear states, which can now be used much more efficiently than before, owing to the state-of-the-art large acceptance superconducting spectrometer, SAMURAI, combined with the new-generation RI beam facility, RIBF, where much higher RI beam intensities are available. Owing to such developments, one can now reach neutron-drip line region for $5 \leq Z \leq \sim 12$. Of particular interest is the issue of the oxygen drip line anomaly. One of the key nucleus beyond the oxygen drip line is ${ }^{26} \mathrm{O}$, whose ground and the first excited state were studied with high precision. The ground state of ${ }^{26} \mathrm{O}$ was found only $18 \pm 3$ (stat) \pm 4 (syst) $\mathrm{keV}$ above the two neutron decay threshold, A such, ${ }^{26} \mathrm{O}$ is the least-energy two-neutron emitter that has ever been observed. It is interesting that such a state could have a long life time, and strong two neutron correlations such as "dineutron".

The next step is to search for the $4 n$ emitter, ${ }^{28} \mathrm{O}$. This nucleus has been called for in terms of the possible doubly magic nucleus, having 8 protons and 20 neutrons. Indeed, more recently at SAMURAI, we measured the decay of ${ }^{28} \mathrm{O}$ produced by the one proton removal of ${ }^{29} \mathrm{~F}$ by the proton target. For that, we introduced the NeuLAND neutron detectors, as well as the thick hydrogen target system MINOS [39, 40]. MINOS has advantages in that one can keep the good energy resolution by detecting the vertex point of the reaction by the tracking of the recoil proton with the TPC (Time Projection Chamber) surrounding the liquid hydrogen target. The analysis is on-going, and in the near future, we hopefully can extract the spectrum of ${ }^{28} \mathrm{O}$.

Not only the oxygen isotopes, but we are planning to measure other exotic nuclei beyond the neutron drip line up to $Z=12$. Such work would shed light on characteristic structures beyond the drip line, such as $2 n, 3 n, 4 n$ correlations, shell evolution across the drip line. We also expect the effects of continuum beyond the drip line to be understood further in the near future.

\section{Acknowledgments}

I thank the collaboration of the day-one campaign experiments. In particular, I thank Y. Kondo, N.A. Orr, J. Gibelin, S. Leblond, Y. Togano, T. Kobayashi, H. Otsu, N. Kobayashi, R. Tanaka, S. Ogoshi, R. Minakata. The present work was supported in part by JSPS KAKENHI Grant No. 24740154, 16H02179, and MEXT KAKENHI Grant No. 24105005.

\section{References}

[1] J. Dobaczewski, N. Michel, W. Nazarewicz, M. Płoszajczak, J. Rotureau, Prog. Part. Nucl. Phys. 59, 432 (2007).

[2] A.B. Migdal, Soviet J. Nucl. Phys. 16, 238 (1973).

[3] M. Matsuo, Phys. Rev. C 73, 044309 (2006).

[4] A. Gezerlis, J. Carlson, Phys. Rev. C 81, 025803 (2010). 
[5] T. Nakamura, et al., Phys. Rev. Lett. 96, 252502 (2006).

[6] H. Sakurai, et al., Phys. Lett. B 448, 180 (1999).

[7] N. Kobayashi, et al., Phys. Rev. C 93, 014613 (2016).

[8] T. Nakamura, et al., Phys. Rev. Lett. 103, 262501 (2009).

[9] T. Nakamura, et al., Phys. Rev. Lett. 112, 142501 (2014).

[10] N. Kobayashi, et al., Phys. Rev. Lett. 112, 242501 (2014).

[11] M. Takechi, et al., Phys. Lett. B 207, 357 (2012).

[12] M. Takechi, et al., Phys. Rev. C 90, 061305(R) (2014).

[13] T. Kobayashi, et al., Nucl. Instr. Methods B 317739 (2013).

[14] T. Baumann, A. Spyrou, M. Thoennessen, Rep. Prog. Phys. 75, 036301 (2012).

[15] T. Aumann, T. Nakamura, Physica Scripta T152, 014012 (2012).

[16] T. Nakamura, et al., Phys. Lett. B 331, 296 (1994).

[17] N. Fukuda, et al., Phys. Rev. C 70, 054606 (2004).

[18] R. Palit, et al., Phys. Rev. C 68, 034318 (2003).

[19] T. Nakamura, et al., Phys. Rev. C 79, 035805 (2009).

[20] T. Nakamura, et al., Phys. Rev. Lett. 83, 1112 (1999).

[21] T. Nakamura, Y. Kondo, Nucl. Instr. Methods B 376156 (2016).

[22] K. Tanaka, et al., Phys. Rev. Lett. 104, 062701 (2010).

[23] N. Kobayashi, et al., Phys. Rev. C 86, 054604 (2012).

[24] Y. Togano, et al., Phys. Lett. B 761, 412 (2016).

[25] J.W. Hwang, et al., Phys. Lett. B 769, 503 (2017).

[26] E. Lunderberg, et al., Phys. Rev. Lett. 108, 142503 (2012).

[27] C.R. Hoffman, et al., Phys. Rev. Lett. 100, 152502 (2008).

[28] C. Caesar, et al., Phys. Rev. C 88, 034313 (2013).

[29] L.V. Grigorenko, I.G. Mukha, C. Scheidenberger, M.V. Zhukov, Phys. Rev. C 84, 021303(R) (2011).

[30] Z. Kohley, et al., Phys. Rev. Lett. 110, 152501 (2013).

[31] L.V. Grigorenko, I.G. Mukha, M.V. Zhukov, Phys. Rev. Lett. 111, 042501 (2013).

[32] Y. Kondo, T. Nakamura et al., Phys. Rev. Lett. 116, 102503 (2016).

[33] K. Hagino, H. Sagawa, Phys. Rev. C 89, 014331 (2014).

[34] K. Hagino, H. Sagawa, Phys. Rev. C 93, 034330 (2016).

[35] Z. Kohley, et al., Phys. Rev. C 91, 034323 (2015).

[36] T. Otsuka, T. Suzuki, J.D. Holt, A. Schwenk, Y. Akaishi, Phys. Rev. Lett. 105, 032501 (2010).

[37] H. Hergert, S. BInder, A. Calci, J. Langhammer, R. Roth, Phys. Rev. Lett. 110, 242501 (2013).

[38] A. Volya, V. Zelevinsky, Phys. At. Nucl. 77, 969 (2014).

[39] A. Obertelli, T. Uesaka, Eur. Phys. A 47, 105 (2011).

[40] A. Obertelli, et al., Eur. Phys. A 50, 8 (2014). 